\title{
Complementing malaria treatment efforts among children in Nigeria: A focus on adequate nutrition
}

\author{
G. O. Ayenigbara
}

Science and Technical Education Department, Adekunle Ajasin University, Akungba-Akoko, Ondo State, Nigeria

Email address:

yenigbarageorge7@yahoo.com

\section{To cite this article:}

G. O. Ayenigbara. Complementing Malaria Treatment Efforts among Children in Nigeria: A Focus on Adequate Nutrition. International Journal of Nutrition and Food Sciences. Vol. 2, No. 4, 2013, pp. 213-216. doi: 10.11648/j.ijnfs.20130204.19

\begin{abstract}
Malaria is by far the most important cause of morbidity and mortality among infants and young children, many of who are also victims of malnutrition, in Nigeria. Malaria is also responsible for a large proportion of neonatal and perinatal mortality. In addition, malaria causes anaemia in children, and undermine their growth and development, and also responsible for low birth weight among infants due to its effects on the foetus, as a result of infection of pregnant mothers in the developing nations, including Nigeria. Malaria is an endemic health problem in Nigeria, and experts affirmed that the co-existence of malnutrition and malaria infection vastly increases the severity of both .Malaria treatment and eradication, particularly among children, is fast becoming a lost battle in Nigeria. This paper therefore recommended the complementary roles of adequate nutrition along with potent drugs use, in malaria treatment for children in Nigeria.
\end{abstract}

Keywords: Malnutrition, Malaria, Anopheles, Children, Nutrients, Water

\section{Introduction}

Human malaria is a disease of wide distribution caused by sporozoa of the genus plasmodium. There are four species of parasites that infect man, they are Plasmodium falciparum, P. vivax, P. malariae, and P. ovale. The arthropod hosts are females of certain species of Anopheles mosquitoes that are vectors for malaria. The burden of disease malaria causes is considerable, amounting to $300-$ 500 million clinical cases per year $-80 \%$ of which occur in Africa [9]. According to [9], malaria is responsible for 1 million deaths per

year - virtually all due to P. falciparum, and $90 \%$ of which are in Africa, including Nigeria. The most common and important infections are those caused by P. falciparum and $P$. vivax, but mixed infections do occur.

Malaria is by far the most important cause of morbidity and mortality in infants and young children. Malaria is also responsible for a large proportion of neonatal and perinatal mortality. In addition, malaria contributes to anaemia in children, undermining their growth and development. Furthermore, malaria is a leading cause of low birth weight among infants through the effects on the foetus, resulting from the infection of pregnant mothers [2], [9]. Apart from these direct effects on child survival, malaria exacerbates poverty, by diminishing productivity and household income, with further negative consequences for children's health and well-being [2].

Clinically, malaria is characterized by fever, hightemperature, hepatomegaly, splenomegaly, varying degrees of anaemia, and various symptoms resulting from involvement of individual organs, convulsions, chills, headache, nausea, vomiting, shock, and death [9], [6], and [11]. Multi-organ failure is common. Death, according to [9] is usually due either to anaemia, usually in children 6 months to 2 years; cerebral malaria in children $2-5$ years; metabolic acidosis in both groups; or a combination of these severe manifestations.

Since the start of malaria control efforts in Nigeria, various attempts have been made to reduce the scourge of this disease. The efforts focused on vector control, using residual insecticide sprays and sanitary inspection [7]. These efforts had limited impact on interrupting the transmission of malaria, because of growing resistance of malaria parasites, (P. falciparum), to insecticides and to drugs for treatment, poverty, ignorance, abundant breeding opportunities for mosquitoes, and dearth of effective drugs [3].

Though a renewed approach to malaria prevention in Nigeria is on going, culminating in the introduction of Insecticide Treated Nets (ITNS), but Ref. [5] found out that in Ekiti, Ogun, Osun and Oyo States, less than 3 percent of 
the respondents slept under ITNS, and the percentages were less than 1 percent in the case of both children under five, and pregnant women. So mosquitoes continued to bite both young and old unabated, with its deleterious health consequences.

Nonetheless, since the consequences of malaria are more pronounced among children, with high morbidity and mortality rates [9], this paper explored the option of ensuring adequate nutrition for a sick child while chemotherapy is on going. When a child is sick, the systems, organs, tissues and cells of the body are all affected negatively. Damaged tissues needed to be repaired, water is needed for rehydration, energy is required to keep the body going, and food ingredients are needed to assist the immune system to withstand pathogens and to aid medication, and thus facilitate and quicken recovery.

Ingredients for body repair, water for rehydration, energy and strength are all supplied through balanced diet. Balanced diet may also assist in building the immune system of the body, which, on its own, may help resist infections or reduce the course of infections. Consequent upon this, 'Ref'. [2] affirmed that when a well-nourished child succumbs to an infection, the period of illness is usually short, the severity of the infection is less, the residual effects are few, and the mortality rate is low. Therefore, provision of adequate nourishment for children becomes imperative before, during, and after malaria infection.

\section{Malnutrition and Infection}

Nutritional deficiencies are factors contributing to the high rate of morbidity, mortality and disabilities in Nigeria. While malnutrition is reported by Ref'. [10], as a direct cause of death in only 2 percent of infants and under-five, it is a contributory factor in a much higher proportion of mortality in these age groups. In the same vein, Reference [4] reported that $35 \%$ of infants mortality are caused by malnourishment worldwide.

Malnutrition is a pathological condition brought about by the inadequacy or over consumption of one or more of the essential nutrients necessary for survival, growth and reproduction, as well as productivity at work [7]. However, Ref. [2] described malnutrition as a state of disease caused by the deficiency or inadequacy, an excess or an imbalance of the supplies of nutrients and or calories.

The multi-factorial causality of diseases becomes very obvious and exemplified in the areas of disease process involving malnutrition and infection. The effects of malnutrition and infection on the human body system and health according to [12] and [13] are synergistic. Synergism is the joint action of agents such that their combined effect is greater than the algebraic sum of their individual effects. This is particularly so in the case of malnutrition and malaria. Malnutrition not only weakens the body's immune system, but aggravates the effect of the diseases in infants, prolongs morbidity, and cause mortality. Malnutrition lowers resistance to infection, and infectious diseases tend to magnify an existing malnutrition [2].

The simultaneous presence of malnutrition and infection results in an interaction with an enlarged effect that is more serious than would be expected if malnutrition or infection acted separately. This may be so when a malnourished child is exposed to malaria. Medicine, according to [2] has long recognized that a nutritional deficiency in the host enhances the risk of an acquired infection and that when the infection sets in, the course and severity of both are greatly enhanced. Thus, infections are more severe in the poorly - nourished while the presence of an infection severely aggravates an existing malnutrition that has played a prominent part in its origin. The consequence of this is that there is an enhanced pathological reaction, in term of numbers of deaths, frequency of complications or duration of disability, far in excess of the expected effect from the two diseases acting independently.

So, the co-existence of malnutrition and infection vastly increases the severity of both. For example, in the poor countries, including Nigeria, the mortality rates from measles were 400 times grater than in some affluent countries, not because of increased virulence of the organism, but because of the co-existence of malnutrition and measles [8], this may also be the case with malaria among children. However, when a well-nourished child succumbs to an infection, like malaria, the period of illness will usually be short, the severity of the infection will be less, the residual effects will be few and the mortality rate will be low.

\section{Malaria and Nutrition in Children}

The body of children requires protein, fats and oil, carbohydrates, Vitamins, mineral salts, and water-about 45 essential nutrients in the correct amount and at the right time. Essential in the sense that their body must get these substances from food because the body is unable to manufacture them at all, or at least not fast enough to meet individuals physiological need [6]. Children must eat foods to obtain the nutrients necessary to keep their bodies growing, and functioning properly. A diet containing adequate amounts of all essential nutrients is vital because various nutrients provide, for example, energy and strength to withstand the rigors of infection, to help build and maintain body tissues damaged by illness, and to help regulate body functions, and thus aid quick recovery.

Some of the relevance of nutrients in the treatment of children with malaria are hereby discussed.

\subsection{Protein}

Malaria infection affects the entire body of the child resulting in organ and tissue damage, and which must be repaired. Therefore, the building blocks of protein, the amino acids, are needed in the form of complete amino acids which are obtained from meat, fish, poultry, eggs, milk, cheese, and soya beans. Other sources are legumes 
and nuts.

However, in Africa, and particularly in Nigeria, where the aforementioned sources may not be obtainable due to poverty, ignorance and superstitious beliefs, people could look inwards to provide these essential amino acids through locally available means. Such means include, for example, insects, which are abundant in Nigeria, and which are known to be rich in protein. Edible insects include grasshoppers, termites, beetles, caterpillars, crickets, honey, locust etc. In addition, are plant sources such as beans, groundnut, melon seeds, locust beans and guinea corn. These protein sources could be factored into the meals of children suffering from malaria to facilitate quick recovery.

\subsection{Fats}

Fats are the most concentrated sources of energy, at 9 calories per gram [6]. Fats help insulate the body and they support, and hold the organs in place, and cushions the organs. So during the prolonged infection of malaria, leading to muscle wasting and emaciation as manifested in sunken eyes, it is the fats that prevents the displacement of organs by holding them in place, particularly, the kidneys and the liver. Fats also help the body to absorb fat-soluble vitamins which are important in recovery, as well as add important flavour and texture to food, a situation that improves appetite and encourage feeding during illness.

However, people are advised to consume monounsaturated fatty acids, such as those found in olive and canola oils, avocados, most nuts, peanuts, safflower oils; and polyunsaturated fat found primarily in fish, corn oil, and soya bean oil, because they are found to protect, and are beneficial to the heart [2], [1] and [6].

\subsection{Carbohydrate (CHO)}

Carbohydrates are needed in the diet primarily to supply energy for body cells. Energy is very essential in coping with the burden of malaria among children. Some cells, such as those found in the brain, and other parts of the nervous system, and in the blood, according to [6], use only carbohydrate for fuel, and when diets lack enough carbohydrates to satisfy the needs of the brain and red blood cells, the body synthesizes carbohydrate from protein. However, in situations of extreme deprivation, when the diet lacks a sufficient among of both carbohydrate and protein, as could be experienced during malaria infection among children, the body turns to its own organs and tissues, breaking down proteins in muscles, the heart, kidneys, and other vital organs to supply carbohydrate needs [6].

This situation may further aggravate and complicate malaria infection, and its treatment among children, and increase morbidity and mortality rates. It becomes very imperative, therefore, to include sufficient quantities of carbohydrate in the diet of malaria patients, particularly among children suffering from malaria.

\subsection{Vitamins}

Vitamins are organic substances required in small amounts to regulate various processes within living cells. Humans need 13 vitamins; 4 are fat - soluble (A, D, E, and $\mathrm{K})$, and 9 are water -soluble (C, and the 8 B-Complex vitamins; thiamin, riboflavin, niacin, Vitamin B-6, folate, Vitamin B-12, biotin, and Pantothenic acid) [6]. Many vitamins help chemical reactions take place. They provide no energy to the body directly, but help unleash the energy stored in carbohydrates, proteins, and fats, thus may be of value to malaria patients [1], [6] and [2].

Vitamins, according to [6] and [1] are critical in the production of red blood cells and the maintenance of the nervous, skeletal, and the immune systems. So, vitamins may help malaria patients to fight anaemia which is a common and serious feature in children, and at the same time help in boosting their immune system so as to effectively resist malaria infection.

Though the body is capable of manufacturing some vitamins, they are abundant in fruits, vegetables, and grains Other sources of vitamins, according to [1], and [2] include milk, eggs, meat, fish, in addition to Vitamins Supplements. Consequently, fruits; vegetables, grains, meat, fish, eggs and milk should be included in the diet of children. Inclusion of vitamins in the diet of children may also prevent vitamin deficiency diseases, the failure of which may worsen malaria infection.

\subsection{Minerals}

Minerals are inorganic elements humans need in relatively small amounts to help regulate body functions, aid in the growth and maintenance of body tissues, and help release energy [9], [6], and [1]. All these functions are indispensable in the treatment of malaria patients and during recuperation from other diseases. There are about 17 essential minerals, but the major ones, those that the body needs include calcium, phosphorus, magnesium, sodium, potassium, and chloride [6]. Also, the essential trace minerals, those that individuals need in minute amounts include copper, fluoride, iodine, iron, selenium, and zink. Sources of minerals include lean meat, dairy products, green leafy vegetables, sweet potatoes, bananas, and other fruits and vegetables; iodine fortified salts, and grains. For effective treatment of children with malaria, these nutrients should be incorporated into their meals.

\subsection{Water}

Water is vital to life, but often ignored. Water is the major component in both foods and the human body. Individual is composed of about $50-60 \%$ water [6]. According to [6], humans can live up to 50 days without food, but only a few days without water. Water is found all over the body, among lean and other tissues, and in blood and other body fluids. Water is used in the digestion and absorption of food, and is the medium in which most of the chemical reactions take place within the body. Some water- 
based fluids, like blood, transport substances around the body, whereas other fluids serve as lubricants or cushions. Water also helps regulate body temperature, thus reducing high temperature, a prominent symptom in malaria infection among children.

Water is contained in almost all foods, particularly in liquids, fruits, and vegetables. The foods and fluids individuals consume provide $80-90 \%$ of daily water intake, the remainder is generated through metabolism. However, water is lost each day in urine, faeces, and sweat, and through evaporation from lungs. But because of the vital role water plays in the body, children who are suffering from malaria should be well hydrated at all times. This could be done by giving them adequate quantities of water to drink, and by placing them on fruits, vegetables, and other diets that can supply water to the body. Also, whenever the temperature is high, large towel may be soaked in cold water, and placed on the body, particularly the head, to bring down the high temperature.

\section{Conclusion and Recommendation}

Sequel to the review of related literature, this paper concludes that the episode of malaria is high in Nigeria, causing high morbidity and mortality rates among children. It has also been documented by experts, [1], [2], and [6], that malnutrition predisposes children to malaria infection, complicates, and prolongs illness, and impede recovery. The paper further concludes that high morbidly and mortality rates from malaria, frequent episode among children, and prolonged illness may not be attributable to inadequate drugs treatment alone. Indeed, inadequate nutrition maybe a factor, so inadequate nutrition should be regarded as inadequate treatment of malaria.

Therefore, this paper recommends that provision of adequate nutrition should be an acceptable practice, along with other therapies, in the prevention, and treatment of malaria, particularly, among children. So, adequate nutrition is a "sine qua non" for effective malaria treatment.

\section{References}

[1] Ajala, J.A. (2006), Understanding Food and Nutrition. Ibadan, MayBest Publications.

[2] Alade, Ibikun Olu (2001), Public Health Nutrition ( $2^{\text {nd }}$ ed) Ilorin, S.O.A. Fosco Ventures Press.

[3] Ayenigbara, G.O. and Boyede, M.M. (2013), Factors Impeding Effective Malaria Prevention in Akoko South East Local Government Area of Ondo State. Unpublished Paper.

[4] British Broadcasting Corporation (BBC) (2013), Comment on Infant Mortality and Malnutrition. $19^{\text {th }}$ May.

[5] Dare, O.O. (2000), "Linking Health to Development: The Oriade Initiative", Takemi Research Paper, Harvard School of Public Health, to be published in Ibadan Achieves of Medicine.

[6] Insel, P.M; and Roth, W.T. (2006), Core Concepts in Health $\left(10^{\text {th }}\right.$ ed) New York. McGraw-Hill.

[7] Hodges, A. (2001), Children's and Women's Right in Nigeria: A Wake-up Call. Publication of National Planning Commission and UNICEF, Nigeria.

[8] Howard, R.B. and Herbold, N.H. (1978), In: Nutrition in Clinical Care, McGraw-Hill Book Co; NY; U.S.A.

[9] Lucas, A.O; and Gilles, H.M. (2003), Short Textbook of Public Health Medicine for the Tropics $\left(4^{\text {th }}\right.$ ed), Book Power.

[10] National Health Management Information System (NHMIS) (1999). Percentage Breakdown of Under-5 Mortality and Morbidity by Reported Causes.

[11] Nordberg, E. (2007), Communicable Diseases, ( $3^{\text {rd }}$ ed), African Medical and Research Foundation (AMREF), Rural Health Series, No. 7.

[12] Scrimshaw, N.S. (1964), Ecological Factors in Nutritional Diseases. Am. J. clin. Nutr. 14: 112-22.

[13] Scrimshaw, N.S. (1964), Causes of Malnutrition. In: G.H. Beaton, ed; Nutrition, A Comprehensive Treatise, Academic Press, New York. 